\title{
Relationship between cortical resection and visual function after occipital lobe epilepsy surgery
}

\author{
${ }^{*}$ Won Heo, MD, ${ }^{1-4}$ June Sic Kim, PhD, ${ }^{5}$ Chun Kee Chung, MD, PhD, ${ }^{1-5}$ and \\ Sang Kun Lee, MD, PhD ${ }^{3,4,6}$
}

\begin{abstract}
Departments of ${ }^{1}$ Neurosurgery and ${ }^{6}$ Neurology, Seoul National University College of Medicine; ${ }^{2}$ Department of Neurosurgery and ${ }^{4}$ Clinical Research Institute, Seoul National University Hospital; ${ }^{3}$ Neuroscience Research Institute, Seoul National University Medical Research Center; and 5Department of Brain and Cognitive Sciences, Seoul National University College of Natural Sciences, Seoul, South Korea
\end{abstract}

OBJECTIVE In this study, the authors investigated long-term clinical and visual outcomes of patients after occipital lobe epilepsy (OLE) surgery and analyzed the relationship between visual cortical resection and visual function after OLE surgery.

METHODS A total of 42 consecutive patients who were diagnosed with OLE and underwent occipital lobe resection between June 1995 and November 2013 were included. Clinical, radiological, and histopathological data were reviewed retrospectively. Seizure outcomes were categorized according to the Engel classification. Visual function after surgery was assessed using the National Eye Institute Visual Functioning Questionnaire 25. The relationship between the resected area of the visual cortex and visual function was demonstrated by multivariate linear regression models.

RESULTS After a mean follow-up period of 102.2 months, 27 (64.3\%) patients were seizure free, and $6(14.3 \%)$ patients had an Engel Class II outcome. Nineteen (57.6\%) of 33 patients had a normal visual field or quadrantanopia after surgery (normal and quadrantanopia groups). Patients in the normal and quadrantanopia groups had better vision-related quality of life than those in the hemianopsia group. The resection of lateral occipital areas 1 and 2 of the occipital lobe was significantly associated with difficulties in general vision, peripheral vision, and vision-specific roles. In addition, the resection of intraparietal sulcus 3 or 4 was significantly associated with decreased social functioning.

CONCLUSIONS The authors found a favorable seizure control rate (Engel Class I or II) of $78.6 \%$, and $57.6 \%$ of the subjects had good visual function (normal vision or quadrantanopia) after OLE surgery. Lateral occipital cortical resection had a significant effect on visual function despite preservation of the visual field.

https://thejns.org/doi/abs/10.3171/2017.5.JNS162963

KEY WORDS occipital lobe epilepsy; clinical outcome; visual field; visual function; resection frequency map

$\mathrm{O}$ CCIPITAL lobe epilepsy (OLE) accounts for a small proportion of extratemporal epilepsies, ${ }^{4}$ and according to previous studies, it constitutes $2 \%-13 \%$ of symptomatic partial epilepsies. ${ }^{2,3,7,16,26}$ Occipital lobe seizures are characterized by visual auras and/or elementary visual hallucinations, ictal blindness, eye blinking, ictal nystagmus, and eye deviation. ${ }^{1,15,19}$ However, determining whether seizures associated with visual aura are of occipital or temporal lobe origin is difficult. ${ }^{3}$ Delineating the seizure-onset zone in the occipital lobe is difficult because of rapid seizure propagation from the occipital lobe to the frontal lobe and adjacent temporal and parietal lobes, as well as to the midbrain tegmentum. ${ }^{6}$ For these reasons, in the absence of occipital lesions found with imaging studies, it can be very difficult to distinguish OLE from other epilepsies. ${ }^{6,18,19,23}$

ABBREVIATIONS AED = antiepileptic drug; $C D$ = cortical dysplasia; EEG = electroencephalography; IPS = intraparietal sulcus; $L O=$ lateral occipital area; $N E I-V F Q-25=$ National Eye Institute Visual Functioning Questionnaire 25; OLE = occipital lobe epilepsy; PPC = posterior parietal cortex; ROI = region of interest.

SUBMITTED November 28, 2016. ACCEPTED May 8, 2017.

INCLUDE WHEN CITING Published online October 27, 2017; DOI: 10.3171/2017.5.JNS162963.

* Drs. Heo and Kim contributed equally to this work. 


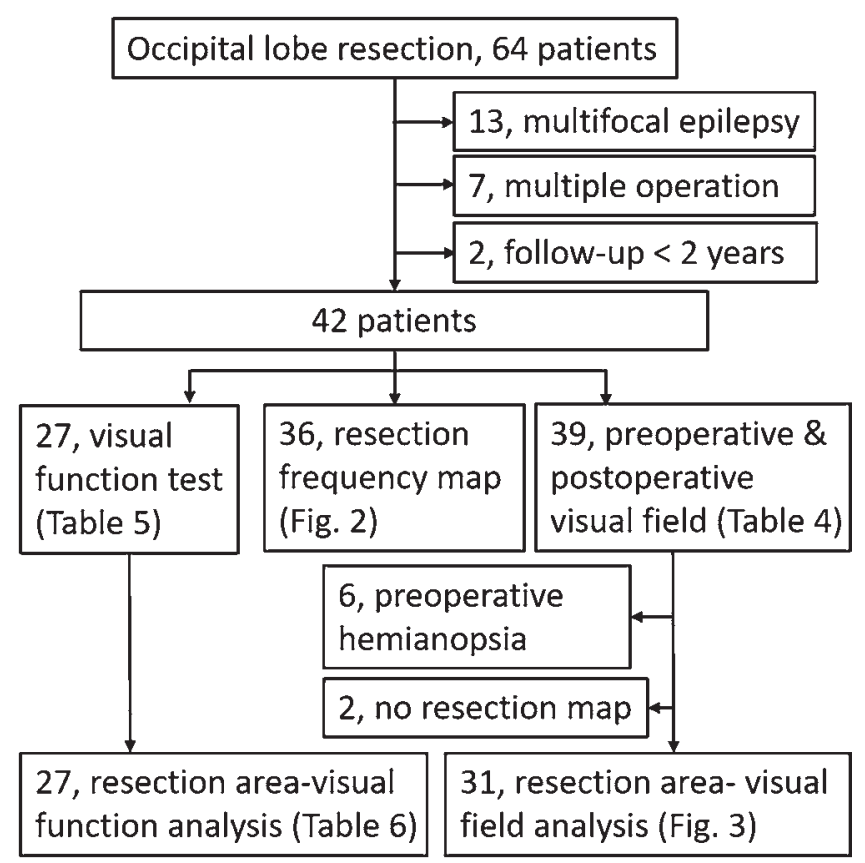

FIG. 1. Analysis flow chart. A total of 1281 epilepsy surgeries, with the exception of invasive monitoring and vagus nerve stimulation, were performed at Seoul National University Hospital between June 1995 and November 2013. Occipital lobe resection was performed in 64 patients. Among them, 13 with multifocal epilepsy were excluded, and 7 who underwent multiple operations were excluded. Two patients were excluded because their follow-up period was less than 2 years. Finally, 42 patients with OLE were included in the study. Preoperative and postoperative MRI scans were available for 36 of the 42 patients; hence, the resection frequency map was restricted to these 36 patients. The results of preoperative and postoperative visual field tests of 39 patients were available. Three other patients were not subjected to visual field tests because they were too young $(2,2$, and 5 years old) to undergo a precise visual field examination. The NEI-VFQ-25 was conducted retrospectively for the study, and 15 people could not be contacted. We received a questionnaire from 27 patients postoperatively. The other 15 patients were excluded from visual function analysis.

Visual field defects, which can occur after OLE surgery, are a considerable problem..$^{4,10,20,23}$ However, studies that qualitatively or quantitatively address visual field defects after OLE surgery have been rare, because OLE is infrequent and not easy to diagnose, and surgical treatment of OLE is rare. Furthermore, visual function is as important an issue as visual field defects. Previous studies categorized human visual cortical areas according to visual function. Probabilistic maps of visual topography have been generated using standard functional MRI paradigms. ${ }^{22}$ We believe that other approaches to sparing visual function are required; thus, it would be meaningful to identify changes in visual function according to visual cortical resection. To our knowledge, confirmation of the relationship between visual function and visual cortical resection has not been attempted in this manner. In this study, we aimed to identify visual field changes after OLE surgery and then evaluate changes in visual function that control the effect of visual field defects.

Therefore, the purposes of this study were to determine the long-term clinical and visual outcomes of OLE sur- gery and to analyze the relationship between visual cortical resection and visual function after OLE surgery.

\section{Methods \\ Patients and Diagnosis}

A total of 42 consecutive patients who were diagnosed with OLE and underwent occipital lobe resection at Seoul National University Hospital between June 1995 and November 2013 were included in the study (Fig. 1). All of the patients had medically intractable epilepsy despite adequate anticonvulsant medication (antiepileptic drugs [AEDs]). The postoperative follow-up duration for each patient was more than 2 years.

The decision to perform surgery was made for patients with the presence of a discrete lesion found with MRI with compatible video electroencephalography (EEG) monitoring or, for patients without a lesion, an ictal-onset zone confirmed by intracranial EEG. In 36 patients, subdural electrodes were implanted to cover the presumed ictal-onset zone according to preoperative evaluations, including video-EEG monitoring. After the electrodes were implanted, video-electrocorticographic monitoring was performed until at least 3 typical seizures occurred. In the other 6 patients, whose lesions were seen with MRI, we performed a lesionectomy when the results of ictal scalp EEG and semiology correlated well with the location of the lesion.

Preoperative and postoperative MR images were available for 36 of the 42 patients included in the study. The resection areas in the remaining 6 patients who had undergone an occipital lobectomy before 1998 were identified through operative records. Use of the resection frequency map was restricted to a subgroup of 36 patients. This study was approved by the institutional review board of the Seoul National University Hospital Clinical Research Institute (Approval H-1506-089-681), and each participant gave written informed consent.

\section{Demographic and Clinical Data}

Demographic and clinical data, such as sex, age at surgery, seizure duration, monthly seizure frequency, seizure semiology, presence of aura, number of preoperative AEDs prescribed, resection area, operation duration, length of hospital stay, and follow-up duration, were reviewed in the electronic medical records. The type of surgery and resection area were identified using postresection MRI. Seizure outcomes after surgery were classified according to the Engel classification method. ${ }^{8}$ The results of visual field tests for 39 patients were available. Goldmann ${ }^{21}$ perimetry was used to test the visual field of 34 patients. The results of visual field testing for the other 5 patients were obtained by confrontation examination. Three other patients were excluded from the analysis of visual field changes because they were too young to undergo precise visual field examination. According to their visual field outcome, we categorized the patients into 1 of 4 groups, normal, quadrantanopia, hemianopia, or other type of visual field defect. Visual function after surgery was assessed in 27 patients using the National Eye Institute Visual Functioning Questionnaire 25 (NEI-VFQ-25). ${ }^{9}, 13$ The other 15 patients were excluded from visual function analysis. 


\section{Preoperative Evaluation}

Multidisciplinary preoperative evaluations, including MRI, FDG-PET, and ictal and interictal SPECT, in addition to video-EEG monitoring, were performed. The purpose of these preoperative studies was to localize an ictalonset zone, and the concordance of these studies enabled delineation of the presumed epileptogenic zone.

\section{Pathological Diagnosis}

Tissue sections from cortical resections were examined histopathologically using routine pathological examination, as previously described. ${ }^{24}$ Tumors were classified according to the revised WHO classification scheme. ${ }^{12}$ The diagnosis of pathological cortical dysplasia (CD) was made according to the grading system developed by Blümcke et al. $^{5}$

\section{Resection Area Map}

T1-weighted 3D spoiled-gradient images with a thickness of $1.0 \mathrm{~mm}$ were used. All resected areas (i.e., regions of interest [ROIs]) were defined manually by comparing each patient's preoperative and postoperative MR images using MRIcro software (available at http://people.cas. sc.edu/rorden/mricro/index.html). In this study, we used preoperative MRI as a template to minimize the distortion of the postoperative MRI. The ROI of each patient was spatially normalized to the Montreal Neurological Institute 305 template. Then, we generated the resection frequency map by combining all the ROIs using MATLAB 7.0 software (MathWorks). ${ }^{11}$ As a result, 2D axial, coronal, and sagittal images and 3D volume-rendering images were obtained using the MRIcro software.

\section{Relationship Between Resection Area and Visual Function}

Individual resection areas were displayed on resection area maps ${ }^{11}$ and then converted to areas in visual topographic probabilistic maps. ${ }^{22}$ We estimated the proportion overlapping with the resection region. The effects on visual function according to the visual cortical resection were analyzed using the Wilcoxon signed-rank test. Every subscale of the NEI-VFQ-25 was analyzed to determine whether a specific visual cortical area was resected. These steps were repeated for each ROI and each questionnaire score. The relationship between the resected area of the visual cortex and visual function was determined by multivariate linear regression models according to each visual function subscale. For each subscale, other cortical resection areas and visual field defects were used as covariates.

\section{Statistical Analysis}

The chi-square and Fisher exact tests were used to analyze binary values, and the Mann-Whitney U-test was used to analyze the continuous values. A binary logistic regression analysis was used to find prognostic factors for a seizure-free outcome. Results of the NEI-VFQ-25 for the hemianopsia, normal, and quadrantanopia visual field groups after OLE surgery were analyzed using the MannWhitney U-test. All statistical analyses were performed using SPSS 21.0 software (SPSS, Inc.). Statistical significance was defined as $\mathrm{p}<0.05$.
TABLE 1. Clinical characteristics

\begin{tabular}{lc}
\hline \multicolumn{1}{c}{ Parameter } & Value \\
\hline Age in yrs (mean [range]) & $21.8 \pm 11.2(2-45)$ \\
\hline Sex (male/female) & $27: 15$ \\
\hline Duration of epilepsy in yrs (mean [range]) & $9.7 \pm 7.1(0.02-27.0)$ \\
\hline Seizure frequency per mo (median [range]) & $4.0(0.17-300.0)$ \\
\hline Mean values are presented with SDs.
\end{tabular}

\section{Results}

\section{Clinical Characteristics}

Forty-two patients with pharmacologically intractable epilepsy underwent occipital lobe resection. Twenty-seven patients were male, and 15 were female. The mean patient age was $21.8 \pm 11.2$ years (range $2-45$ years). The mean duration of epilepsy was $9.7 \pm 7.1$ years (range 0.02-27.0 years), and the median seizure frequency was $4.0 /$ month (range 0.17-300.0/month) (Table 1).

\section{Auras and Initial Seizure Semiology}

Twenty-nine of the 42 patients experienced at least 1 type of aura, and 7 patients had experienced multiple types of aura. Elementary visual hallucination as an aura was reported most frequently (16 [38.1\%] patients). Headache and dizziness constituted the second most common aura (8 [19.0\%] patients). The third most frequent aura was visual illusion (4 [9.5\%] patients), followed by blindness or visual field defect (3 [7.1\%] patients). Autonomic and psychic auras were observed in 3 and 2 patients, respectively.

\section{Seizure Semiology}

An early alteration of awareness without significant motor activity (dialeptic seizure) was noted in $16(38.1 \%)$ patients, automotor seizure was present in $11(26.2 \%)$, and versive seizure was present in $7(16.7 \%)$. Thirteen patients exhibited secondarily generalized tonic-clonic seizures, and $8(19.0 \%)$ patients had predominantly generalized tonic-clonic seizures.

\section{Surgery}

Occipital lobectomy was performed in 15 patients. Among them, 14 patients underwent their operation before 2000, and 1 patient underwent surgery in 2003. Pathological findings included CD in all patients. No definite lesion was visible in the MR images of 15 patients. The other 27 patients underwent focal occipital resection. Gross-total resection was performed in cases of tumorous conditions and encephalomalacia. The mean operation time was $271.2 \pm 89.8$ minutes (range 150-560 minutes). The mean hospital stay was $11.8 \pm 11.4$ days (range $4-60$ days). The mean duration of follow-up after surgery was $102.2 \pm 62.9$ months (range 25-244 months). A cumulative occipital lobe resection frequency map was created using MRIcro software (Fig. 2).

\section{Seizure Outcomes}

After a mean follow-up time of 102.2 months (range 25-244 months), 33 (78.6\%) of the 42 patients experienced a good clinical outcome after OLE surgery; 27 (64.3\%) 


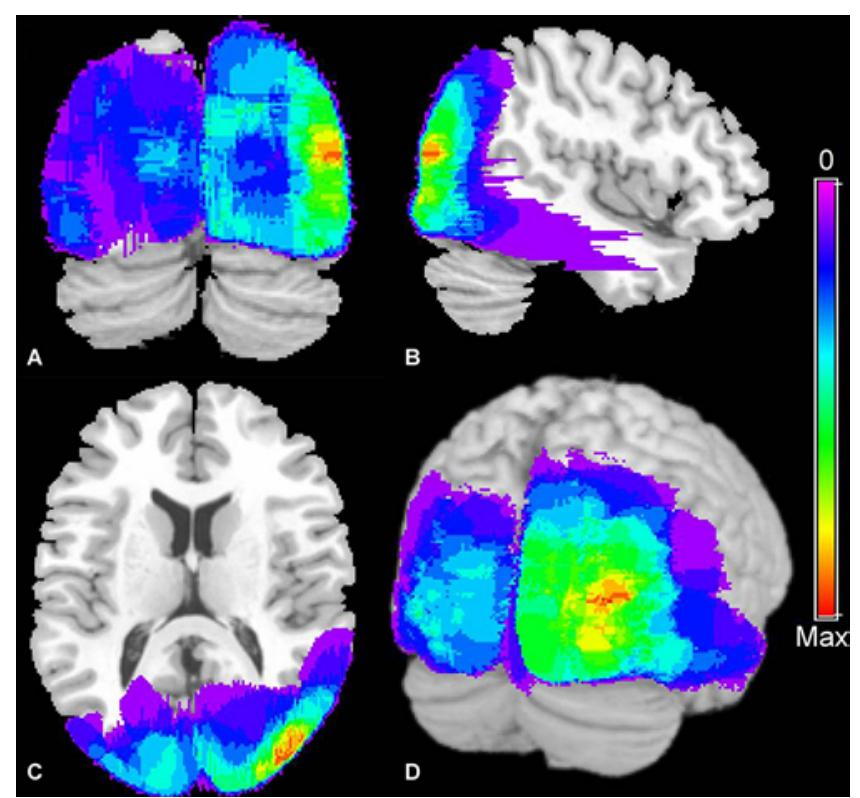

FIG. 2. Cumulative occipital lobe resection frequency map. Resected areas were delineated in 36 patients using preoperative and postoperative MR images. Each resection area was stacked onto the template. The colored areas depict the resected areas, and the color indicates the frequency of resection: coronal (A), sagittal (B), axial (C), and 3D-rendered (D) images. Most frequently resected was the right lateral temporal area.

patients became seizure free (Engel Class I), and $6(14.3 \%)$ patients had a favorable outcome (Engel Class II). Five patients reached Engel Class III, and 4 patients reached Engel Class IV (Table 2).

\section{Pathological Examination}

Histopathological examinations revealed CD in 32 patients $(76.2 \%)$, dysembryoplastic neuroepithelial tumor in $4(9.5 \%)$, encephalomalacia in $2(4.8 \%)$, and extraventricular neurocytoma, gangliocytoma, ganglioglioma, and an unclassifiable atypical glioneuronal tumor in 1 patient each $(2.4 \%)$.

\section{Seizure Outcome-Associated Factors}

A logistic regression test was used to identify prognostic factors for a seizure-free outcome. Factors with a $\mathrm{p}$ value of less than 0.25 in a univariate analysis were included in a multivariate analysis to control for multicollinearity. The following factors were considered for the multivariate analysis: duration of epilepsy, seizure frequency, preoperative AEDs, presence of visual aura, and pathological findings. Age and sex were not correlated with a seizure-free outcome $(p>0.05)$. In the multivariate analysis, seizure frequency $(\mathrm{p}=0.041$; OR 0.971; 95\% CI 0.944-0.999) and preoperative AEDs (especially more than 4 [ $\mathrm{p}=0.035$; OR $0.039 ; 95 \%$ CI 0.002-0.800]) were significantly associated with poor seizure outcome. In addition, seizure outcomes were analyzed according to the extent of resection. However, we found no significant difference in outcomes between patients who underwent lobectomy and those who underwent focal resection $(\mathrm{p}=$ 0.076) (Table 3).
TABLE 2. Seizure outcomes

\begin{tabular}{lc}
\hline \multicolumn{1}{c}{ Engel Classification } & No. of Patients (\%) \\
\hline I, seizure free & $27(64.3)$ \\
\hline II, rare seizures & $6(14.3)$ \\
\hline III, 90\% seizure reduction & $5(11.9)$ \\
\hline IV, no change & $4(9.52)$ \\
\hline
\end{tabular}

\section{Visual Field Changes After Surgery}

A visual field test was performed for 39 patients before and after surgery. Before surgery, 29 patients had a normal visual field, 4 had quadrantanopia, 5 had hemianopsia, and 1 had irregularly shaped visual field defects. After surgery, 9 patients maintained a normal visual field, 10 had quadrantanopia, 18 had hemianopsia, and 2 had irregularly shaped visual field defects. Visual field defects increased or were newly developed in 24 patients after surgery; 20 patients with previously normal vision developed visual defects after surgery, and 4 patients with preexisting quadrantanopia developed complete hemianopsia. Nine patients did not exhibit any visual field defects before or after occipital lobe resection, and 6 patients with a previous visual field defect experienced no change in the defect after occipital resection (Table 4).

We analyzed the relationship between the visual field defects according to occipital resection area. Of the 36 patients whose resected area was identified using preoperative and postoperative MRI, we included 31 patients who had a normal or quadrantanopic visual field before surgery. The V1v, V2v, V1d, V2d, and V3d areas of resection were strongly associated with visual field defects after surgery $(\mathrm{p}=0.017, \mathrm{p}<0.001, \mathrm{p}=0.017, \mathrm{p}=0.006$, and $\mathrm{p}=$ 0.003 , respectively, according to the Fisher exact test). The patients were categorized into 1 of 2 groups according to the change in visual field defects after surgery, and 2 resection frequency maps were drawn (Fig. 3).

We compared the visual field changes according to the pathological findings for 33 patients who had a normal visual field or quadrantanopia before surgery. For the patients with $\mathrm{CD}$, the rate of postoperative hemianopsia was higher than that for the patients with a tumorous condition. However, we found no significant difference between the 2 groups $(p=0.670)$. In addition, the visual field changes were analyzed according to the location of the lesion. For the patients with a medially located lesion, the rate of postoperative hemianopsia was higher than that for the patients with another lesion location. In addition, a significant difference between the 2 groups was found ( $\mathrm{p}=0.038$ ).

The NEI-VFQ-25 was administered postoperatively to 27 patients. The NEI-VFQ-25 scores of the hemianopsia group $(n=14)$ and the normal and quadrantanopia groups $(n=13)$ were compared after surgery. The difference between the total scores of the groups was significant (76.0 [hemianopsia group] vs 89.7 [normal-or-quadrantanopia group] $[\mathrm{p}=0.029])$. Scores of the 3 groups for general, near, and distance vision, social functioning, and mental health also were significantly different $(\mathrm{p}<0.05)$. However, scores for general health, vision-specific role difficulties, dependency, driving, peripheral vision, and ocular 
TABLE 3. Prognostic factors for seizure-free outcome

\begin{tabular}{|c|c|c|c|c|}
\hline \multirow[b]{2}{*}{ Factor } & \multicolumn{2}{|c|}{ Seizure-Free Outcome } & \multirow{2}{*}{$\begin{array}{c}\mathrm{p} \\
\text { Value }\end{array}$} & \multirow{2}{*}{$\begin{array}{c}\text { Adjusted OR }(95 \% \mathrm{Cl}) \\
\text { p Value }\end{array}$} \\
\hline & Yes $(n=27)$ & No $(n=15)$ & & \\
\hline Age at op in yrs (median [min, max]) & $21.0(4.0,45.0)$ & $20.0(2.0,41.0)$ & 0.875 & \\
\hline $\operatorname{Sex}(M / F)$ & $19: 8$ & $8: 7$ & 0.270 & \\
\hline Duration of epilepsy in yrs (median [min, max]) & $8.0(0.02,22)$ & $11(1,27)$ & 0.103 & \\
\hline Seizure frequency per mo (median [min, max]) & $3(0.17,150)$ & $7(2,300)$ & 0.031 & 0.971 (0.944-0.999), 0.041 \\
\hline Preop AEDs (no.) & & & 0.020 & 0.050 \\
\hline 1 & 7 & 1 & & \\
\hline 2 & 7 & 4 & & \\
\hline 3 & 10 & 2 & & \\
\hline$>4$ & 3 & 8 & & $0.039(0.002-0.800), 0.035$ \\
\hline Presence of visual aura (no. [\%]) & $16(59.3)$ & $5(33.3)$ & 0.107 & \\
\hline Pathology (CD) (no. [\%]) & $18(66.7)$ & $14(93.3)$ & 0.068 & $0.065(0.004-1.166), 0.063$ \\
\hline
\end{tabular}

pain were not significantly different between the 3 groups $(p>0.05)$ (Table 5).

We compared visual function after surgery according to pathological findings for these 27 patients. For the patients with $\mathrm{CD}$, the visual function scores were lower than those for the patients with a tumorous condition. A significant difference between the 2 groups was found ( $p$ $=0.034)$. In addition, visual function after surgery was analyzed according to the location of the lesion. For the patients with a medially located lesion, the visual function scores were lower than those for the patients with another lesion location. However, we found no significant difference between the 2 groups $(\mathrm{p}=0.683)$.

\section{Multivariate Linear Regression Models for Finding Relationships Between the Visual Cortical Resection Area and Visual Function Scores Without the Effect of Visual Field Defects}

General vision scores decreased by 18.6 points in accord with lateral occipital area $2(\mathrm{LO} 2)$ resection $(\mathrm{p}=0.043$; adjusted B -18.608; 95\% CI -36.583 to -0.634 ), peripheral vision was decreased by 23.5 points in accord with LO1 resection ( $\mathrm{p}=0.001$; adjusted $\mathrm{B}-23.505 ; 95 \% \mathrm{CI}-36.601$ to -10.409 ), social functioning was decreased by 25.0 points in accord with intraparietal sulcus 3 (IPS3) or IPS4 resection $(\mathrm{p}=0.028$; adjusted $\mathrm{B}-25.000 ; 95 \% \mathrm{CI}-47.048$ to -2.952 ), and vision-specific role difficulties were decreased by 18.2 points in accord with LO1 resection ( $\mathrm{p}=$ 0.022 ; adjusted B $-18.199 ; 95 \%$ CI -35.508 to -2.890$)$. In the dorsal-lateral area of the occipital lobe, LO1 was associated with peripheral vision and vision-specific role difficulties, and LO2 was associated with general vision. IPS3 or IPS4 was associated with social functioning (Table 6).

\section{Discussion}

OLE is uncommon. The aims of our study were to determine the long-term clinical and visual outcomes of OLE surgery and analyze the relationship between visual cortical resection and visual function. In this study, 42 consecutive patients who underwent OLE surgery at a single institution between June 1995 and November 2013 were included. This study includes one of the largest series to have focused on surgical treatment for OLE., ${ }^{1,4,15,19}$

In this study, 27 (64.3\%) patients became seizure free, and another $6(14.3 \%)$ patients entered the rare-seizure state. Nine $(21.4 \%)$ of the 42 patients achieved Engel Class III or IV, which is similar to results in previous studies., ${ }^{4,15}$ However, only a few studies have assessed prognostic factors for seizure-free outcomes. ${ }^{1}$ We analyzed the prognostic factors for a seizure-free outcome using binary logistic regression testing, and we determined that seizure frequency and preoperative AEDs, particularly more than 4, were significantly associated with poor seizure outcomes in a multivariate analysis $(\mathrm{p}<0.05)$. After surgery, 56.3\% (18 of 32) of the patients with a pathological diagnosis of CD became seizure free, and a 90\% (9 of 10) seizure-free rate in patients with another pathological finding, such as a tumor, was found. The pathological examination result was another factor associated with a seizure-free outcome; however, this factor was not statistically significant $(\mathrm{p}=$ 0.063; OR 0.065; 95\% CI 0.004-1.166). This result corresponds with those of previous studies. ${ }^{1,26}$

One of the most difficult problems in the surgical treatment for OLE is the new development or aggravation of preexisting visual field defects. In this study, visual field defects were newly developed or increased in $24(61.5 \%)$

TABLE 4. Visual field outcomes

\begin{tabular}{lcc}
\hline \multirow{2}{*}{$\begin{array}{c}\text { Visual Field } \\
(\mathrm{N}=39)\end{array}$} & \multicolumn{2}{c}{ No. of Patients } \\
\cline { 2 - 3 } & Before Surgery & After Surgery \\
\hline Normal & 29 & 9 \\
\hline Quadrantanopia & 4 & 10 \\
\hline Hemianopsia & 5 & 18 \\
\hline Other types of visual defect & 1 & 2 \\
\hline Change in the visual field & & \\
\hline Normal to normal & - & 9 \\
\hline Normal to defect & - & 20 \\
\hline Increased defect & - & 4 \\
\hline No change in defect & - & 6 \\
\hline
\end{tabular}



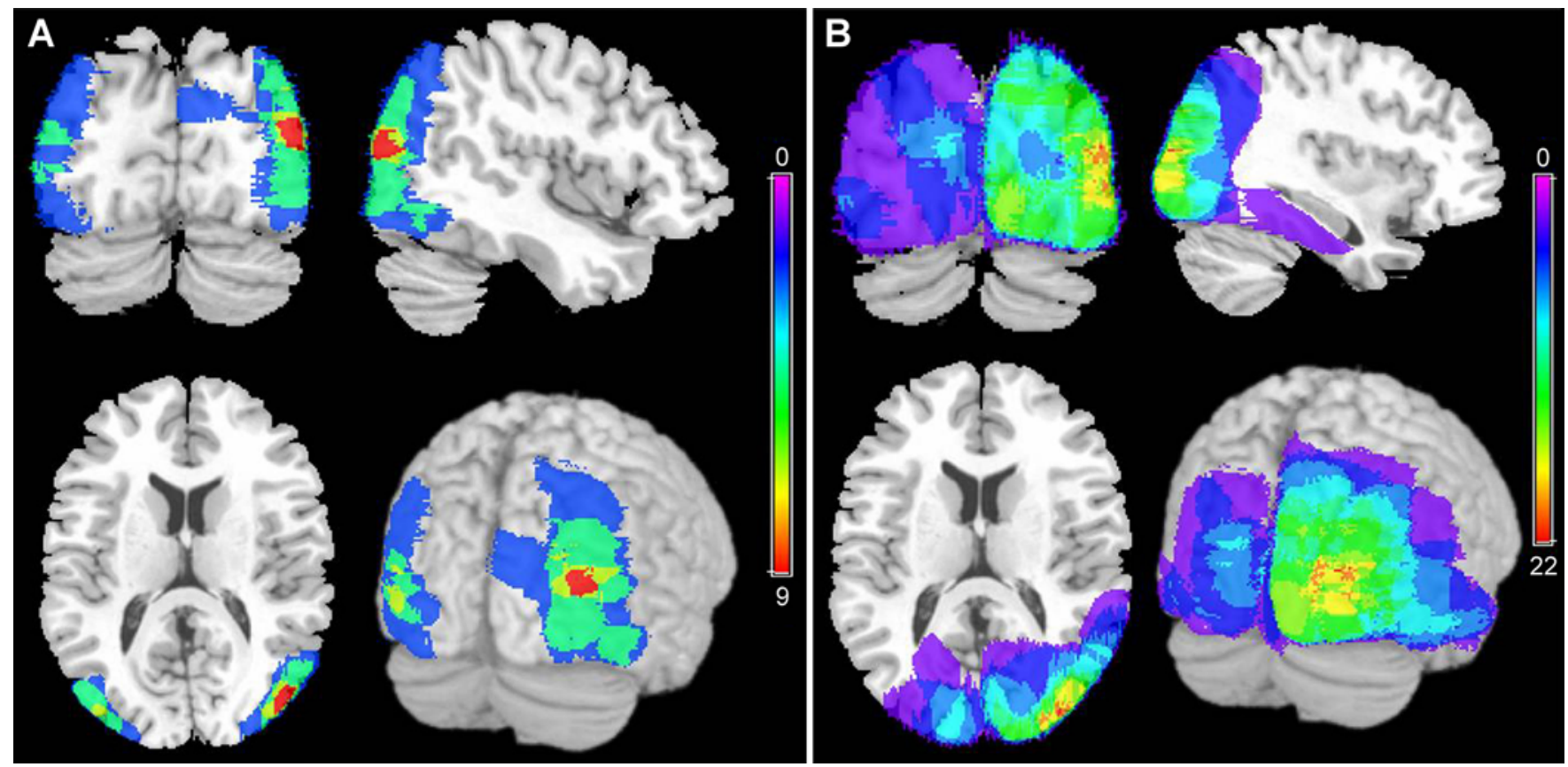

FIG. 3. Resection frequency map according to visual field change after surgery. 2D axial, coronal, and sagittal images and 3D volume-rendered images were obtained using MRIcro software. Of the 36 patients whose resected area was identified using preoperative and postoperative MR images, 31 who had a normal or quadrantanopic visual field before surgery were included. Nine patients had an intact visual field and 22 patients had a worsened visual field after occipital lobe resection. Each of the 2 resection frequency maps was generated according to the visual field changes after surgery. Because the number of patients in the 2 groups was different, the frequency was divided by the number of patients to compare the 2 groups visually. A: Intact visual field after occipital lobe resection. Bar $=9$ cases. B: Worsened visual field after occipital lobe resection. Bar $=22$ cases.

of 39 patients, which is not significantly different from the results of previous studies. ${ }^{4,6,10,25}$ In a previous study, visual field deficits were rare in patients with lateral OLE compared with those with medial OLE. ${ }^{23}$ In this study, we analyzed the relationship between visual field defects and occipital area resection in 33 patients with a normal or quadrantanopic visual field before surgery. As a result, resection in primary visual cortical areas, such as the V1v, $\mathrm{V} 2 \mathrm{v}, \mathrm{V} 1 \mathrm{~d}, \mathrm{~V} 2 \mathrm{~d}$, and V3d areas, was strongly associated with visual field defects. According to the resection maps (Fig. 2), this finding is supported visually by our results. In previous studies, visual field defects after OLE surgery

TABLE 5. NEI-VFQ-25 scores after OLE surgery

\begin{tabular}{lcccc}
\hline \multirow{2}{*}{ NEI-VFQ-25 Subscale } & \multicolumn{3}{c}{ Mean NEI-VFQ-25 Score $(95 \% \mathrm{Cl})$} & $\begin{array}{c}\mathrm{p} \\
\text { Value }\end{array}$ \\
\cline { 2 - 4 } General health & Hemianopsia* $(\mathrm{n}=14)$ Normal or Quadrantanopia* $(\mathrm{n}=13)$ & Total & $71.3(56.6-86.0)$ & 0.325 \\
\hline General vision & $64.3(73.3-85.3)$ & $78.8(55.9-101.8)$ & $73.3(64.0-82.6)$ & $0.019 \dagger$ \\
\hline Near vision & $62.9(51.0-74.7)$ & $84.6(71.4-97.8)$ & $76.5(68.4-84.7)$ & $0.017 \dagger$ \\
\hline Distance vision & $67.3(55.4-79.1)$ & $86.5(77.0-96.1)$ & $78.2(70.9-85.6)$ & $0.007 \dagger$ \\
\hline Driving & $69.3(58.9-79.7)$ & $87.8(79.2-96.4)$ & $63.9(46.6-81.1)$ & 0.200 \\
\hline Peripheral vision & $52.7(28.8-76.7)$ & $75.0(39.1-1108)$ & $81.7(75.0-88.5)$ & 0.118 \\
\hline Color vision & $76.8(67.9-85.7)$ & $87.5(76.8-98.2)$ & $93.0(88.3-97.7)$ & 0.110 \\
\hline Ocular pain & $88.5(80.6-96.3)$ & $97.9(93.3-102.5)$ & $96.8(93.5-100.0)$ & 0.220 \\
\hline Vision-specific role difficulties & $93.8(87.6-99.9)$ & & $81.5(72.7-90.3)$ & 0.155 \\
\hline Dependency & $75.0(60.3-89.7)$ & $88.5(78.5-98.4)$ & $85.2(77.0-93.3)$ & 0.094 \\
\hline Social functioning & $79.1(66.2-92.1)$ & $91.7(81.4-101.9)$ & $90.5(85.2-95.7)$ & $0.021 \dagger$ \\
\hline Mental health & $84.8(77.3-92.4)$ & $92.7(92.7-102.8)$ & $81.9(73.9-90.0)$ & $0.017 \dagger$ \\
\hline Total & $74.1(62.4-85.7)$ & $90.4(79.7-101.0)$ & $82.6(76.4-88.8)$ & $0.029 \dagger$ \\
\hline
\end{tabular}

* Status of postoperative visual field.

$\dagger p<0.05$. 
TABLE 6. Multivariate linear regression models for finding relationships between visual cortical resection area and visual function scores

\begin{tabular}{|c|c|c|c|c|c|}
\hline NEI-VFQ Subscale $(n=27)$ & Resection Area* & Unadjusted B (95\% Cl) & p Value & Adjusted B $(95 \% \mathrm{Cl})$ & p Value \\
\hline General vision & LO2 & $-20.000(-38.421$ to -1.579$)$ & 0.0345 & $-18.608(-36.583$ to -0.634$)$ & $0.0431 \dagger$ \\
\hline Peripheral vision & L01 & $-15.000(-27.666$ to -2.334$)$ & 0.0222 & $-23.505(-36.601$ to -10.409$)$ & $0.0013+$ \\
\hline \multirow{3}{*}{ Social functioning } & TO2 & $-14.732(-27.852$ to -1.613$)$ & 0.0294 & & \\
\hline & IPS3 & $-29.167(-53.363$ to -4.970$)$ & 0.0203 & $-25.000(-47.048$ to -2.952$)$ & $0.0282 \dagger$ \\
\hline & IPS4 & $-29.167(-53.363$ to -4.970$)$ & 0.0203 & $-25.000(-47.048$ to -2.952$)$ & $0.0282 \dagger$ \\
\hline Mental health & LO2 & $-17.706(-33.598$ to -1.813$)$ & 0.0304 & $-15.136(-31.318$ to 1.046$)$ & 0.0653 \\
\hline \multirow{2}{*}{$\begin{array}{l}\text { Vision-specific role dif- } \\
\text { ficulties }\end{array}$} & L01 & $-22.206(-38.476$ to -5.936$)$ & 0.0095 & $-18.199(-33.508$ to -2.890$)$ & $0.0220 \dagger$ \\
\hline & LO2 & $-22.222(-39.009$ to -5.435$)$ & 0.0115 & & \\
\hline \multirow[t]{2}{*}{ Dependency } & L01 & $-17.513(-33.177$ to -1.849$)$ & 0.0299 & & \\
\hline & LO2 & $-18.067(-34.089$ to -2.045$)$ & 0.0286 & $-14.303(-30.747$ to 2.142$)$ & 0.0850 \\
\hline
\end{tabular}

Data are regression coefficients (B) and $95 \%$ confidence intervals.

* LO1 and LO2 are located in the lateral occipital cortex anterior and lateral to V3d and posterior to V5/MT+; LO2 is anterior to LO1. TO2 is identified in the lateraltemporal cortex along the medial-temporal gyrus, anterior to LO2. IPS3 and IPS4 are located in the anterior-lateral branch of the IPS and are separated by an upper vertical meridian.

$\dagger p<0.05$.

were mentioned. However, visual function using the NEIVFQ-25 in patients after OLE surgery has not been assessed. With the results of the NEI-VFQ-25, we could assess social functioning and mental health, as well as visual function. In our study, the scores of the questionnaire were analyzed according to the visual field defects after surgery. The scores for the driving and vision-associated subscales were lower than those for the life-associated subscales, such as vision-specific role difficulties, dependency, social functioning, and mental health, in all of the patients. After surgery, the scores of patients in the hemianopsia group were lower than those of patients in the normal and quadrantanopia groups, and significant differences were found in several subscales, such as general, near, and distance vision, social functioning, and mental health $(\mathrm{p}<$ 0.05 ). Among 33 patients who had normal vision or quadrantanopia before surgery, 9 patients had a normal visual field and 10 patients had quadrantanopia after surgery. We could assume that these $19(57.6 \%)$ patients might have good visual function after OLE surgery.

In this study, we analyzed the relationship between each visual cortical resection and each NEI-VFQ-25 score using visual topographic probabilistic maps. ${ }^{22}$ Because visual functions can be influenced by visual field defects, these defects were controlled as a covariate. We found some statistically significant relationships $(\mathrm{p}<0.05)$. In the dorsallateral areas of the occipital lobe, LO1 was significantly correlated with peripheral vision and vision-specific role difficulties, and LO2 was significantly correlated with general vision. Among the parietal-frontal areas, IPS3 or IPS4 was significantly correlated with social functioning according to the multivariate linear regression models.

LO1 and LO2 are parts of the lateral occipital cortex, which is located in the fundus of the lateral (middle) occipital sulcus; LO2 is anterior to LO1. In a previous study, the topography, stimulus selectivity, and anatomical locations of LO1 and LO2 indicated that these areas integrate shape information from multiple visual submodalities in retinotopic coordinates. The retinotopic and functional properties of LO1 and LO2 suggest that these areas cor- respond to the 2 visual areas unique to humans that lack exact homologs in the macaque visual cortex. The functional properties of LO1 and LO2 suggest that they play different but complementary roles in shape recognition. ${ }^{17}$ In our study, the resection of LO1 and LO2 was correlated significantly with peripheral vision, vision-specific role difficulties, and general vision. These results agree well with those of previous studies.

According to a previous study, IPS3 and IPS4, located between IPS2 and IPS5 and superior to parietal lobule 1 (SPL1), lack exact homologs in the macaque visual cortex. Therefore, the functional homologies for human IPS3 and IPS4 remain unknown. IPS1, IPS2, and the medial SPL1 prefer saccadic eye movements. In contrast, IPS 3 and IPS4 displayed responses during the performance of smoothpursuit eye movements that were significantly stronger than those during saccadic eye movements. Thus, it is possible that IPS3 and IPS4 are human-specific areas that evolved from disproportional enlargement of the posterior parietal cortex (PPC), which resulted in a distribution of functions that is broader than that in the monkey PPC. ${ }^{14}$ In this study, the resection of IPS3 or IPS4 was correlated significantly with social functioning. These findings are important, because ours is the first trial to have identified the relationships between specific visual cortical areas and specific visual functions using resection in OLE.

There are several limitations in this study to consider. First, it was a retrospective study; thus, some data from radiological and visual field examinations and visual function tests were missing. For patients who underwent occipital lobectomy before 1998, no MR images were available for us to use to analyze the relationships between the resected areas of the visual cortex and visual function scores. Therefore, most of the patients whose data were included in the relationship analysis underwent focal resection after 2000. The majority of focal resections were performed in the dorsal-lateral occipital lobe. These factors could have influenced the results of the analysis. Second, the study period was long. Since 1994, the senior author (C.K.C.) performed half of all the surgeries. Five other 
neurosurgeons contributed the other half. The senior author (C.K.C.) established the surgical protocol, which was followed by others. Therefore, there was little variation in the surgical procedure regardless of who performed it. We found no statistically significant differences in Engel classification grades or visual field changes according to surgeon $(\mathrm{p}=0.747$ and 0.180 , respectively, Pearson chisquare test). We also found no statistically significant differences in postoperative visual function score according to surgeon ( $p=0.422$, Mann-Whitney U-test). In addition, magnetoencephalography was introduced and was used to localize epileptiform brain activity in 2005. In addition, 3.0-T MRI was introduced in 2009. With the development of diagnostic methods, it has become easier to localize epileptogenic zones. Third, this study was limited by its small sample size, although it is one of the largest studies of surgical treatment for OLE to date. A large prospective randomized study could provide more information regarding the relationship between the visual cortical resection area and visual function. Fourth, other limitations of this study include the inherent technical difficulties that arise from the manual delineation of each resected area on a map. To minimize these difficulties, an experienced neurosurgeon (W.H.) delineated all of the ROIs manually, and another experienced neurosurgeon (C.K.C.) examined each case in detail. Last, we could not compare preoperative and postoperative NEI-VFQ-25 scores, because the questionnaire was not administered before surgery.

\section{Conclusions}

We found a $78.6 \%$ favorable seizure control rate (Engel Class I or II), and $57.6 \%$ of the patients had good visual function (normal or quadrantanopia) after OLE surgery. These results suggest that surgery could play a significant role in patients with medically intractable OLE. In addition, the results of this study have important implications, because it is the first trial to have identified the relationship between specific visual cortical areas and visual function using resection in OLE.

\section{Acknowledgments}

We appreciate the statistical advice from the Medical Research Collaborating Center at the Seoul National University Hospital and the Seoul National University College of Medicine. This research was supported by the Basic Science Research Program through the National Research Foundation of Korea (NRF), funded by the Ministry of Science, ICT and Future Planning (Grant 2014R1A2A1A11049662).

\section{References}

1. Aykut-Bingol C, Bronen RA, Kim JH, Spencer DD, Spencer SS: Surgical outcome in occipital lobe epilepsy: implications for pathophysiology. Ann Neurol 44:60-69, 1998

2. Bidziński J, Bacia T, Ruzikowski E: The results of the surgical treatment of occipital lobe epilepsy. Acta Neurochir (Wien) 114:128-130, 1992

3. Bien CG, Benninger FO, Urbach H, Schramm J, Kurthen M, Elger CE: Localizing value of epileptic visual auras. Brain 123:244-253, 2000

4. Binder DK, Von Lehe M, Kral T, Bien CG, Urbach H, Sch- ramm J, et al: Surgical treatment of occipital lobe epilepsy. J Neurosurg 109:57-69, 2008

5. Blümcke I, Thom M, Aronica E, Armstrong DD, Vinters HV, Palmini A, et al: The clinicopathologic spectrum of focal cortical dysplasias: a consensus classification proposed by an ad hoc Task Force of the ILAE Diagnostic Methods Commission. Epilepsia 52:158-174, 2011

6. Blume WT, Whiting SE, Girvin JP: Epilepsy surgery in the posterior cortex. Ann Neurol 29:638-645, 1991

7. Boesebeck F, Schulz R, May T, Ebner A: Lateralizing semiology predicts the seizure outcome after epilepsy surgery in the posterior cortex. Brain 125:2320-2331, 2002

8. Engel J Jr, Van Ness PC, Rasmussen TB, Ojemann LM: Outcome with respect to epileptic seizures, in Engel J Jr (ed): Surgical Treatment of the Epilepsies, ed 2. New York: Raven Press, 1993, pp 609-621

9. Heo JW, Yoon HS, Shin JP, Moon SW, Chin HS, Kwak HW: A validation and reliability study of the Korean version of National Eye Institute Visual Function Questionnaire 25. J Korean Ophthalmol Soc 51:1354-1367, 2010

10. Jobst BC, Williamson PD, Thadani VM, Gilbert KL, Holmes GL, Morse RP, et al: Intractable occipital lobe epilepsy: clinical characteristics and surgical treatment. Epilepsia 51:2334-2337, 2010

11. Kim YH, Kim CH, Kim JS, Lee SK, Chung CK: Resection frequency map after awake resective surgery for non-lesional neocortical epilepsy involving eloquent areas. Acta Neurochir (Wien) 153:1739-1749, 2011

12. Kleihues P, Sobin LH: World Health Organization classification of tumors. Cancer 88:2887-2887, 2000

13. Klein R, Moss SE, Klein BEK, Gutierrez P, Mangione CM: The NEI-VFQ-25 in people with long-term type 1 diabetes mellitus: the Wisconsin Epidemiologic Study of Diabetic Retinopathy. Arch Ophthalmol 119:733-740, 2001

14. Konen CS, Kastner S: Representation of eye movements and stimulus motion in topographically organized areas of human posterior parietal cortex. J Neurosci 28:8361-8375, 2008

15. Kun Lee S, Young Lee S, Kim DW, Soo Lee D, Chung CK: Occipital lobe epilepsy: clinical characteristics, surgical outcome, and role of diagnostic modalities. Epilepsia 46:688695,2005

16. Kuzniecky R, Gilliam F, Morawetz R, Faught E, Palmer C, Black L: Occipital lobe developmental malformations and epilepsy: clinical spectrum, treatment, and outcome. Epilepsia 38:175-181, 1997

17. Larsson J, Heeger DJ: Two retinotopic visual areas in human lateral occipital cortex. J Neurosci 26:13128-13142, 2006

18. Palmini A, Andermann F, Olivier A, Tampieri D, Robitaille Y: Focal neuronal migration disorders and intractable partial epilepsy: results of surgical treatment. Ann Neurol 30:750757, 1991

19. Salanova V, Andermann F, Olivier A, Rasmussen T, Quesney LF: Occipital lobe epilepsy: electroclinical manifestations, electrocorticography, cortical stimulation and outcome in 42 patients treated between 1930 and 1991. Surgery of occipital lobe epilepsy. Brain 115:1655-1680, 1992

20. Tandon N, Alexopoulos AV, Warbel A, Najm IM, Bingaman WE: Occipital epilepsy: spatial categorization and surgical management. J Neurosurg 110:306-318, 2009

21. Trope GE, Britton R: A comparison of Goldmann and Humphrey automated perimetry in patients with glaucoma. Br J Ophthalmol 71:489-493, 1987

22. Wang L, Mruczek RE, Arcaro MJ, Kastner S: Probabilistic maps of visual topography in human cortex. Cereb Cortex 25:3911-3931, 2015

23. Williamson PD, Thadani VM, Darcey TM, Spencer DD, Spencer SS, Mattson RH: Occipital lobe epilepsy: clinical characteristics, seizure spread patterns, and results of surgery. Ann Neurol 31:3-13, 1992 
24. Wolf HK, Zentner J, Hufnagel A, Campos MG, Schramm J, Elger CE, et al: Surgical pathology of chronic epileptic seizure disorders: experience with 63 specimens from extratemporal corticectomies, lobectomies and functional hemispherectomies. Acta Neuropathol 86:466-472, 1993

25. Yang PF, Jia YZ, Lin Q, Mei Z, Chen ZQ, Zheng ZY, et al: Intractable occipital lobe epilepsy: clinical characteristics, surgical treatment, and a systematic review of the literature. Acta Neurochir (Wien) 157:63-75, 2015

26. Yun CH, Lee SK, Lee SY, Kim KK, Jeong SW, Chung CK: Prognostic factors in neocortical epilepsy surgery: multivariate analysis. Epilepsia 47:574-579, 2006

\section{Disclosures}

The authors report no conflict of interest concerning the materi- als or methods used in this study or the findings specified in this paper.

\section{Author Contributions}

Conception and design: Chung. Acquisition of data: Heo, Lee. Analysis and interpretation of data: Heo, Kim. Drafting the article: Heo. Critically revising the article: Chung, Heo. Reviewed submitted version of manuscript: Chung, Lee. Approved the final version of the manuscript on behalf of all authors: Chung. Statistical analysis: Heo, Kim. Administrative/technical/material support: Kim. Study supervision: Chung, Lee.

\section{Correspondence}

Chun Kee Chung, Department of Neurosurgery, Seoul National University College of Medicine, 101 Daehak-ro, Jongno-gu, Seoul 110-744, South Korea. email: chungc@snu.ac.kr. 